\title{
Management of obesity and cardiometabolic risk - role of phentermine/extended release topiramate
}

This article was published in the following Dove Press journal: Diabetes, Metabolic Syndrome and Obesity:Targets and Therapy 12 February 2014

Number of times this article has been viewed

\author{
Arianne N Sweeting' \\ Eddy Tabet ${ }^{\prime}$ \\ Ian D Caterson ${ }^{1,2}$ \\ Tania P Markovic ${ }^{1,2}$ \\ 'Department of Endocrinology, \\ Royal Prince Alfred Hospital, \\ Sydney, NSW, Australia; ${ }^{2}$ Boden \\ Institute of Obesity, Nutrition, \\ Exercise \& Eating Disorders, \\ University of Sydney, \\ NSW, Australia
}

\begin{abstract}
The US Food and Drug Administration (FDA) recently approved lorcaserin and the combination of phentermine and extended release topiramate (phentermine/topiramate ER) for the treatment of obesity in conjunction with a lifestyle intervention, expanding the therapeutic options for long-term obesity pharmacotherapy, which was previously limited to orlistat. Combination phentermine/topiramate ER is associated with greater weight loss compared to its constituent monotherapy, with a more favorable adverse effect profile. Phentermine/topiramate ER also appears to have beneficial effects on cardiometabolic risk, although longer-term cardiovascular safety data are required. While there are no head-to-head studies among the currently available obesity pharmacotherapy agents, phentermine/topiramate ER appears to have a superior weight loss profile. This review will discuss the epidemiology, natural history, and cardiometabolic risk associated with obesity, provide an overview on current obesity pharmacotherapy, and summarize the recent clinical efficacy and safety data underpinning the FDA's approval of both phentermine/topiramate ER and lorcaserin as pharmacotherapy for a long-term obesity intervention.
\end{abstract}

Keywords: obesity, phentermine/topiramate extended release, safety and efficacy, review

\section{Introduction}

\section{Epidemiology and the natural history of obesity}

Obesity is a chronic disease that has become a serious global threat to public health, with the worldwide prevalence of obesity exponentially increasing. Recent World Health Organization data reveal that $35 \%$ of adults are overweight and $11 \%$ of adults are obese, corresponding to an estimated 1.46 billion overweight and 500 million obese adults, respectively. ${ }^{1}$ This equates to almost double the incidence of obesity in 1980, while the age-standardized mean global body mass index (BMI) increased by $0.4-0.5 \mathrm{~kg} / \mathrm{m}^{2}$ per decade in both men and women. ${ }^{1}$

The US now accounts for the highest global incidence of overweight and obesity, particularly in men, with the respective lifetime risk of approximately $50 \%$ and $25 \%{ }^{2}$ Future projections indicate that if the current US trajectory is not curbed, by 2030, close to half of all men and women will be obese. ${ }^{3}$ Despite these alarming statistics, only one-third of obese patients receive an obesity diagnosis or weight-related treatment advice from their physicians. ${ }^{4}$

Department of Endocrinology,

Level 6 Royal Prince Alfred Hospital,

Camperdown Sydney, NSW,

Australia 2050

Tel +6I 95157226

Email Arianne.Sweeting@sswahs.nsw. gov.au

\section{Cardiometabolic sequelae of obesity}

Obesity is caused by an interaction of environmental factors, genetic predisposition, and human behavior that confers increased risk of morbidity and mortality predominantly 
through cardiometabolic sequelae. ${ }^{5}$ Evidence suggests that obesity is implicated in close to 3 million deaths worldwide every year. ${ }^{6}$ Overweight and obese patients are at an increased risk of developing cardiometabolic complications including hypertension, type 2 diabetes mellitus, dyslipidemia, and cardiovascular diseases, as well as conditions such as osteoarthritis, obstructive sleep apnea, hepatobiliary diseases, and certain malignancies. ${ }^{1}$

Weight reduction of 5\%-10\% from baseline is associated with improvements in cardiometabolic risk, with consequent reductions in obesity-related morbidity and mortality rates, ${ }^{7,8}$ providing the rationale for interventions that target even modest weight loss.

The poor history of obesity pharmacotherapy illustrates the critical importance of long-term cardiovascular safety data in the risk-benefit analysis of newer agents. Fenfluramine, dexfenfluramine, combination fenfluramine/ phentermine, and sibutramine have all been withdrawn from the market because of significant cardiovascular safety concerns, including increased risk of nonfatal myocardial infarction and nonfatal stroke (sibutramine), ${ }^{9}$ primary pulmonary hypertension (combination fenfluramine/phentermine), ${ }^{10}$ and valvular regurgitation (fenfluramine, dexfenfluramine). ${ }^{11}$ Nevertheless, analysis of the major trials of failed pharmacotherapy, such as the Sibutramine Cardiovascular Outcomes (SCOUT) study, ${ }^{9}$ are invaluable in minimizing adverse outcomes with obesity pharmacotherapy by identifying the most appropriate patient population in which to use these agents. For example, the SCOUT study demonstrated that only patients with pre-existing cardiovascular disease had an increased risk of new cardiovascular events, and thus newer pharmacotherapy may be unsafe in patients with significant comorbidities and must be used with caution. ${ }^{9}$ Interpretation of the SCOUT data also suggests that the long-term continuation of therapy, despite not achieving weight loss, may have confounded the results. Thus, pharmacotherapy should be ceased if patients do not achieve $>5 \%$ weight loss in order to minimize the long-term risks of therapy. ${ }^{12}$

\section{Review of current pharmacotherapy available for obesity: efficacy, safety, and tolerability including impact on comorbidities}

Prior to the US Food and Drug Administration (FDA) approval of lorcaserin and phentermine/topiramate extended release (ER), the only FDA-approved long-term obesity pharmacotherapy was orlistat; the latter remains the only long-term therapy approved in Europe. These therapies are approved as an adjunct to lifestyle interventions for the treatment of overweight adults with comorbidities, and of obese patients with or without comorbidities. ${ }^{20}$

Four monotherapies have been approved for the shortterm ( $\leq 3$ months) management of obesity: phentermine; diethylpropion; benzphetamine; and phendimetrazine. ${ }^{13}$ Only phentermine and diethylpropion are available in the US.

\section{Phentermine}

The noradrenergic sympathomimetic amine agent, phentermine, was approved by the FDA in 1959 for short-term ( $\leq 12$ weeks) obesity treatment, in addition to lifestyle interventions, in obese or overweight adults with obesityrelated comorbidities. It is the most commonly prescribed appetite suppressant in the US. While the exact mechanism of action is unknown, phentermine acts centrally to induce appetite suppression through the upregulation of dopamine, noradrenaline, and serotonin activity, and it has also been proposed to increase energy expenditure. ${ }^{14,15}$

Prior to the introduction of phentermine/topiramate ER, phentermine was the most effective obesity pharmacotherapy for both weight reduction and improvement in a number of comorbidities, particularly waist circumference and lipid profiles. ${ }^{16}$ A meta-analysis of six placebo-controlled randomized trials published between 1975 and 1999 of 2-24 weeks duration found that phentermine therapy was associated with a $3.6 \mathrm{~kg}$ (confidence interval [CI]: 0.6-6.0 kg) greater weight loss compared with placebo. ${ }^{17}$

Safety concerns for phentermine relate primarily to its cardiovascular risk factor profile, which is associated with phentermine's stimulant properties, and are dose-dependent. Long-term clinical trials also indicate that there is increased tolerance and dependency associated with prolonged use. ${ }^{18}$ The most common adverse events, typical of sympathomimetic amines, include dry mouth and insomnia, ${ }^{19}$ which were generally self-limiting and mild-moderate in clinical trials. More serious complications include palpitations, tachycardia, and hypertension. Moreover phentermine has been associated with cardiovascular complications including primary pulmonary hypertension, cardiovascular disease, and cardiovascular or cerebrovascular events, and it is contraindicated in individuals with these conditions, in those with psychiatric disorders, including anorexia or depression, or in those at risk of drug dependency. ${ }^{20}$ 


\section{Topiramate}

Topiramate is a sulfamate-substituted monosaccharide approved by the FDA in 1996 for the treatment of seizure disorders, and for migraine prophylaxis in 2004. It is associated with significant weight loss and maintenance; however, it is not approved for obesity monotherapy owing to its adverse effects profile, which is dose related. The specific mechanism for topiramate associated weight loss is unclear; however, it does mediate appetite suppression, satiety, and leads to a reduction in addictive food craving by antagonizing excitatory voltage-gated sodium and calcium channels, $\alpha$-amino-3-hydroxy-5-methyl-4-isoxazolepropionic acid and kainite receptors, and it also modifies the taste of food by inhibiting carbonic anhydrase isoenzymes. ${ }^{21,22}$ Animal studies also implicate increased energy expenditure by augmenting the activity of the inhibitory neurotransmitter $\gamma$-aminobutyric acid. ${ }^{19,22,23}$ Furthermore, topiramate appears to attenuate the adverse metabolic sequelae associated with obesity, independent of its weight loss properties, by enhancing insulin action, glucose transport, and adiponectin in adipocytes, skeletal muscle, and pancreatic beta cells..$^{24,25}$

A meta-analysis of six studies assessing the efficacy of topiramate for weight loss over 24 weeks found that the percentage weight loss in topiramate-treated individuals was 6.5\% (CI: 4.8\%-8.3\%). ${ }^{17} \mathrm{~A}$ sensitivity analysis assuming no weight loss among individuals lost to follow-up demonstrated a $3.6 \%$ mean weight loss with topiramate (CI: $2.6-4.8 \%) .{ }^{17}$ The degree of topiramate-associated weight loss appears to correspond to baseline body weight (ie, obese individuals lost a higher percentage of body weight compared to normal or overweight individuals). ${ }^{26}$

Despite topiramate's proven efficacy, its high incidence of adverse effects has prevented its use as an obesity monotherapy. Dose-dependent neuropsychiatric and cognitive adverse effects have been reported, ${ }^{27,28}$ while its effects on carbonic anhydrase inhibition can lead to paresthesia and other complications associated with metabolic acidosis. ${ }^{46,47}$ Rarely, topiramate is associated with vision disturbances, the most serious of which are severe acute myopia and secondary angle-closure glaucoma.

\section{Orlistat}

Orlistat is a pancreatic lipase inhibitor that reduces the metabolism and thus the absorption of some dietary fats by binding to lipase in the gut lumen, inhibiting $\sim 30 \%$ of fat absorption. It is the only obesity therapy currently approved in both Europe and the US for the long-term treatment of obesity, and it remains the only obesity therapy with longterm safety and efficacy data.

Orlistat appears to be modestly effective in promoting weight loss, and thus although it is indicated for the longterm treatment of obesity, weight loss maintenance remains difficult. Accordingly, attrition rates average $33 \%$ in studies. ${ }^{29}$ A Cochrane meta-analysis of randomized controlled trials showed an average greater weight reduction of $2.9 \%$ in patients who received orlistat as an adjunct to lifestyle modification (CI: $2.5 \%-3.4 \%$ ) compared to placebo, with equivalent weight regain in both groups over time. ${ }^{30} \mathrm{~A}$ meta-analysis of 22 studies of orlistat over 12 months demonstrated a $2.89 \mathrm{~kg}$ (CI: $2.27-3.51 \mathrm{~kg}$ ) associated weight loss compared to placebo, with total weight loss in the orlistat-treated patients of $8.13 \mathrm{~kg} .{ }^{17}$

Orlistat also appears to have beneficial effects on metabolic parameters. A 4-year Swedish randomized controlled trial of 3,305 nondiabetic obese patients showed that orlistat therapy resulted in modest weight loss compared to placebo (5.8 $\mathrm{kg}$ versus $3.0 \mathrm{~kg}$ ), and it reduced the risk of new onset diabetes mellitus by $37.3 \%{ }^{31}$ Orlistat has also been associated with improved lipid, independent of weight loss, and blood pressure parameters. ${ }^{31,32}$ The clinical utility of orlistat is limited predominantly by its gastrointestinal side effects; however, these are generally mild and mitigated on a lowfat diet. ${ }^{30}$

\section{Lorcaserin}

Lorcaserin is a selective serotonin $2 \mathrm{C}$ receptor agonist that acts within the hypothalamus to decrease food intake by promoting increased satiety. This is mediated via release of melanotropin- $\alpha$, which stimulates melanocortin receptor-4, resulting in appetite satiety. ${ }^{33}$ Three 1-year randomized, placebo-controlled Phase III trials have demonstrated that lorcaserin is associated with moderate weight loss in conjunction with lifestyle interventions. ${ }^{34-36}$

The 2-year Behavioral Modification and Lorcaserin for Obesity and Overweight Management in Diabetes Mellitus ${ }^{34}$ (BLOOM) trial evaluated 3,182 overweight or obese individuals (with $\geq 1$ comorbidity, excluding diabetes), and demonstrated significant weight loss in the lorcaserin versus placebo group after 1 year (mean weight loss: $-5.8 \mathrm{~kg}$ and $-2.2 \mathrm{~kg}$, respectively). Overall, lorcaserin was associated with a $3.6 \%$ placebo-subtracted weight reduction after 1 year $(5.8 \%$ weight loss with lorcaserin versus $2.2 \%$ for placebo). After 1 year, $47.5 \%$ of individuals receiving lorcaserin achieved 
$\geq 5 \%$ weight loss and $22.6 \%$ achieved $\geq 10 \%$ weight loss compared to $20.3 \%$ and $7.7 \%$, respectively, of those receiving placebo. After 2 years, although weight increased among individuals on lorcaserin therapy, long-term therapy was associated with lower mean body weight than either placebo or those receiving lorcaserin in year 1 followed by placebo. ${ }^{34}$ The Behavioral Modification and Lorcaserin Second Study for Obesity Management ${ }^{35}$ (BLOSSOM) trial evaluated the efficacy of lorcaserin in 4,008 obese and overweight patients with obesity-related complications. It was found that lorcaserin was associated with a significantly greater mean percent weight loss compared with placebo $(-2.8 \%,-4.7 \%$, and $-5.8 \%$ for placebo, lorcaserin $10 \mathrm{mg}$ daily, and lorcaserin $10 \mathrm{mg}$ twice daily, respectively).

The effects of lorcaserin in 604 obese individuals with diabetes on therapy with variable glycemic control (hemoglobin $\mathrm{A}_{1 \mathrm{c}}\left[\mathrm{HbA}_{1 \mathrm{c}}\right]$ 7.0\%-10.0\%) were specifically evaluated in the Behavioral Modification and Lorcaserin for Obesity and Overweight Management in Diabetes Mellitus (BLOOM-DM) study. ${ }^{36}$ Almost $50 \%$ of individuals treated with lorcaserin achieved an $\mathrm{HbA}_{1 \mathrm{c}}<7.0 \%$, which correlated with the degree of weight loss achieved. However, these improvements in cardiometabolic risk were not durable. Although glycemic and lipid parameters appeared to be more favorable when compared with placebo in the first year of study, they were no longer significant by the end of the 2 years, reflecting the effects of weight regain. ${ }^{36}$

Lorcaserin is generally well tolerated, with the most common adverse effects associated with its use including headache, dizziness, fatigue, and nausea. Although lorcaserin has a similar mechanism of action to fenfluramine and dexfenfluramine, its receptor specificity may attenuate the risk of valvulopathy or cardiovascular sequelae, as echocardiogram studies in the major clinical trials of lorcaserin were within normal range. ${ }^{34,35}$ However, there is concern that the studies were not adequately powered to detect possible cardiovascular adverse effects, owing to the lower than expected event rate. ${ }^{37}$

\section{Role of combination phenterminel topiramate ER and comparative efficacy for obesity}

Combination phentermine/topiramate ER was approved by the FDA in July 2012 for the long-term treatment of obesity in obese or overweight individuals with obesity related co-morbidities at a recommended dose of 7.5/46 $\mathrm{mg}$ daily, in conjunction with lifestyle intervention. It has not been approved in Europe primarily due to the lack of long-term data on cardiovascular safety outcomes.

\section{Efficacy}

Combination pharmacotherapy for obesity aims to achieve greater overall weight loss by targeting multiple complementary pathways that regulate energy homeostasis, to overcome physiological counter-regulatory mechanisms that frequently lead to weight regain. It also allows lower and therefore better tolerated doses of the therapy's components to be used. Although there are no head-to-head studies, phentermine/ topiramate ER currently appears to be the most effective available obesity pharmacotherapy, although longer-term data are required.

Five recent key clinical trials have evaluated the efficacy, safety, and cardiometabolic effects of phentermine/topiramate ER in conjunction with lifestyle modifications. The superiority of combination pharmacotherapy was established in the 28-week randomized controlled Phase III EQUATE study. ${ }^{38,39}$ Obese patients ( $\mathrm{n}=756$ ) (BMI: $30-45 \mathrm{~kg} / \mathrm{m}^{2}$ ) were randomized to one of seven treatment arms (phentermine/topiramate ER mid-dose [7.5/46 mg] or full-dose [15/92 mg], phentermine $7.5 \mathrm{mg}$ or $15 \mathrm{mg}$, topiramate $46 \mathrm{mg}$ or $92 \mathrm{mg}$, and a placebo arm). Both doses of phentermine/topiramate ER significantly reduced mean body weight compared to an equivalent monotherapy or placebo. Mid- and full-dose phentermine/topiramate ER was associated with mean reductions in weight of $8.5 \%$ and $9.2 \%$, respectively, compared to $1.7 \%$ for placebo, $6.1 \%$ for full-dose phentermine, and $6.4 \%$ for full-dose topiramate. ${ }^{38}$

The 1-year EQUIP study randomized 1,287 participants with a $\mathrm{BMI} \geq 35 \mathrm{~kg} / \mathrm{m}^{2}$ without significant comorbidities to either placebo, low-dose (3.75/23 mg), or high-dose (15/92 mg) phentermine/topiramate ER in conjunction with a lifestyle intervention. ${ }^{41}$ Phentermine/topiramate ER was associated with significantly greater weight reductions from baseline, with a mean body weight decrease of $10.9 \%$ with full-dose phentermine/topiramate ER, 5.1\% with low-dose phentermine/topiramate ER, versus $1.6 \%$ with placebo. Overall, phentermine/topiramate ER produced a $9.4 \%$ placebo-subtracted weight reduction after 1 year. Significantly more participants on phentermine/topiramate ER achieved $\geq 5 \%$ and $\geq 10 \%$ weight loss compared to placebo: $66.7 \%$ and $47.2 \%$ on the full dose, $44.9 \%$ and $18.8 \%$ on the low dose, versus $17.3 \%$ and $7.4 \%$ for placebo, respectively. ${ }^{41}$

The largest of these trials, CONQUER, assessed the safety, efficacy and impact on the cardiometabolic sequelae of obesity of phentermine/topiramate ER in 2,487 overweight and obese participants (BMI: $27-45 \mathrm{~kg} / \mathrm{m}^{2}$ ) with two or more obesity-related comorbidities. Participants were randomized to placebo, mid-dose $(7.5 / 46 \mathrm{mg})$, or high-dose $(15 / 92 \mathrm{mg})$ phentermine/topiramate ER over the course of 
56 weeks. ${ }^{27}$ Phentermine/topiramate ER was associated with significantly greater weight loss $(-1.4 \mathrm{~kg}$ for placebo, -8.1 $\mathrm{kg}$ for mid-dose, and $-10.2 \mathrm{~kg}$ for high-dose therapy), as well as greater maintenance of weight loss. Mean weight loss from baseline in the placebo, mid-, and full-dose groups was $1.2 \%, 7.8 \%$, and $9.8 \%$, respectively. Weight loss of $\geq 5 \%$ and $\geq 10 \%$, respectively, occurred in $21 \%$ and $7 \%$ of individuals in the placebo arm, $62 \%$ and $37 \%$ in the mid-dose arm, and $70 \%$ and $48 \%$ in the high-dose arm. ${ }^{27}$

The SEQUEL study demonstrated the durability of weight loss maintenance with phentermine/topiramate ER in obese individuals with comorbidities. SEQUEL was a 2-year extension study, enrolling 676 subjects who had participated in the CONQUER trial for an additional 52 weeks, maintaining their original randomized treatment assignment. ${ }^{42}$ Six hundred and seventy-six of a total 866 eligible participants continued in the extension study, with $84 \%$ completing the study overall. Weight gain occurred in all groups; however, the mean weight loss at the end of 2 years was greater with both mid$(-9.3 \%)$ and high-dose $(-10.5 \%)$ phentermine/topiramate ER versus $-1.8 \%$ for the placebo group. In addition, $79.3 \%$ of participants in the high-dose and $75.2 \%$ in the mid-dose phentermine/topiramate ER groups achieved $\mathrm{a} \geq 5 \%$ reduction in mean body weight from baseline, compared to $30 \%$ in the placebo group, while $53.9 \%$ of participants in the middose and $50.3 \%$ in the high-dose phentermine/topiramate ER groups achieved $\geq 10 \%$ weight loss. ${ }^{42}$ The impact of selection bias must be taken into account in interpreting these results, as the study cohort in the SEQUEL trial consisted only of patients who completed the CONQUER study, and these patients were presumably responders and well motivated.

\section{Cardiometabolic parameters}

The majority of morbidity and mortality outcomes in obesity relate to complications from cardiometabolic disease, and even modest weight reductions and decreased waist circumference are associated with improvements in several cardiometabolic parameters, including systemic arterial pressure, lipid profile and blood glucose levels. ${ }^{8}$ As phentermine/topiramate ER appears to be a potent obesity pharmacotherapy, any subsequently achieved weight loss would be expected to have a favorable impact on cardiometabolic risk factors, with the caveat of the known adverse cardiovascular risk profile associated with its constituent monotherapies.

Treatment with phentermine/topiramate ER is associated with favorable blood pressure and lipid profiles, as summarized in Table 1. The EQUIP study demonstrated significant reductions in both systolic and diastolic blood pressure, and an improved lipid profile among those individuals on high-dose phentermine/topiramate ER, although this study group was characterized by a relative lack of obesity-related comorbidities. ${ }^{41}$ The CONQUER trial also confirmed significant improvements in systolic blood pressure, triglycerides, and high-density lipoprotein cholesterol with both doses of phentermine/topiramate ER, in addition to small, but statistically significant, reductions in diastolic blood pressure and low-density lipoprotein cholesterol with full-dose phentermine/topiramate ER. ${ }^{27}$ The SEQUEL trial demonstrated the durability of improvements in cardiometabolic disease with ongoing reductions in systolic and diastolic blood pressure, necessitating a decrease in antihypertensive therapies, as well as sustained improvements in glycemic control and lipid profiles. ${ }^{42}$

The CONQUER trial demonstrated significant improvements in relation to glycemic profiles. ${ }^{27}$ Participants with diabetes mellitus treated with phentermine/topiramate ER had greater improvements in glycemic parameters compared to placebo after 1 year, with significant reductions in $\mathrm{HbA}_{1 \mathrm{c}}$, fasting blood glucose, and insulin levels following an oral glucose tolerance test. Only $1.5 \%$ and $0.6 \%$ of participants with diabetes mellitus in the mid- and high-dose phentermine/ topiramate ER groups, respectively, required an escalation in oral hypoglycemic medication compared to $12 \%$ in the placebo group. There was also less progression to diabetes mellitus in the phentermine/topiramate ER group, with the annualized incidence rate for the development of diabetes at $9.0 \%$ for placebo, $6.7 \%$ for mid-dose, and $4.4 \%$ for highdose phentermine/topiramate ER. ${ }^{27}$ A 1-year randomized controlled trial of full-dose phentermine/topiramate ER in 130 obese subjects with diabetes mellitus on either dietcontrol or oral hypoglycemic agents demonstrated an $\mathrm{HbA}_{1 \mathrm{c}}$ reduction of $1.6 \%$ (versus $1.1 \%$ in the placebo group), correlating with increased use of oral hypoglycemic agents in the placebo group versus an overall decrease in the phentermine/ topiramate ER group. ${ }^{42}$

The effect of phentermine/topiramate ER on obstructive sleep apnea has also been assessed in a small single-center trial over 28 weeks, demonstrating an improved apneahypopnea index in the treatment arm compared to placebo. The reduction in body weight correlated positively with improvements in the apnea-hypopnea index score. ${ }^{43}$

\section{Safety and tolerability}

Combination pharmacotherapy is theoretically associated with improved tolerability through lower doses of constituent monotherapy which, in turn, enables sustained weight loss 
Table I Comparison of efficacy and safety of orlistat, lorcaserin, and phentermine/topiramate ER for weight loss and cardiometabolic parameters

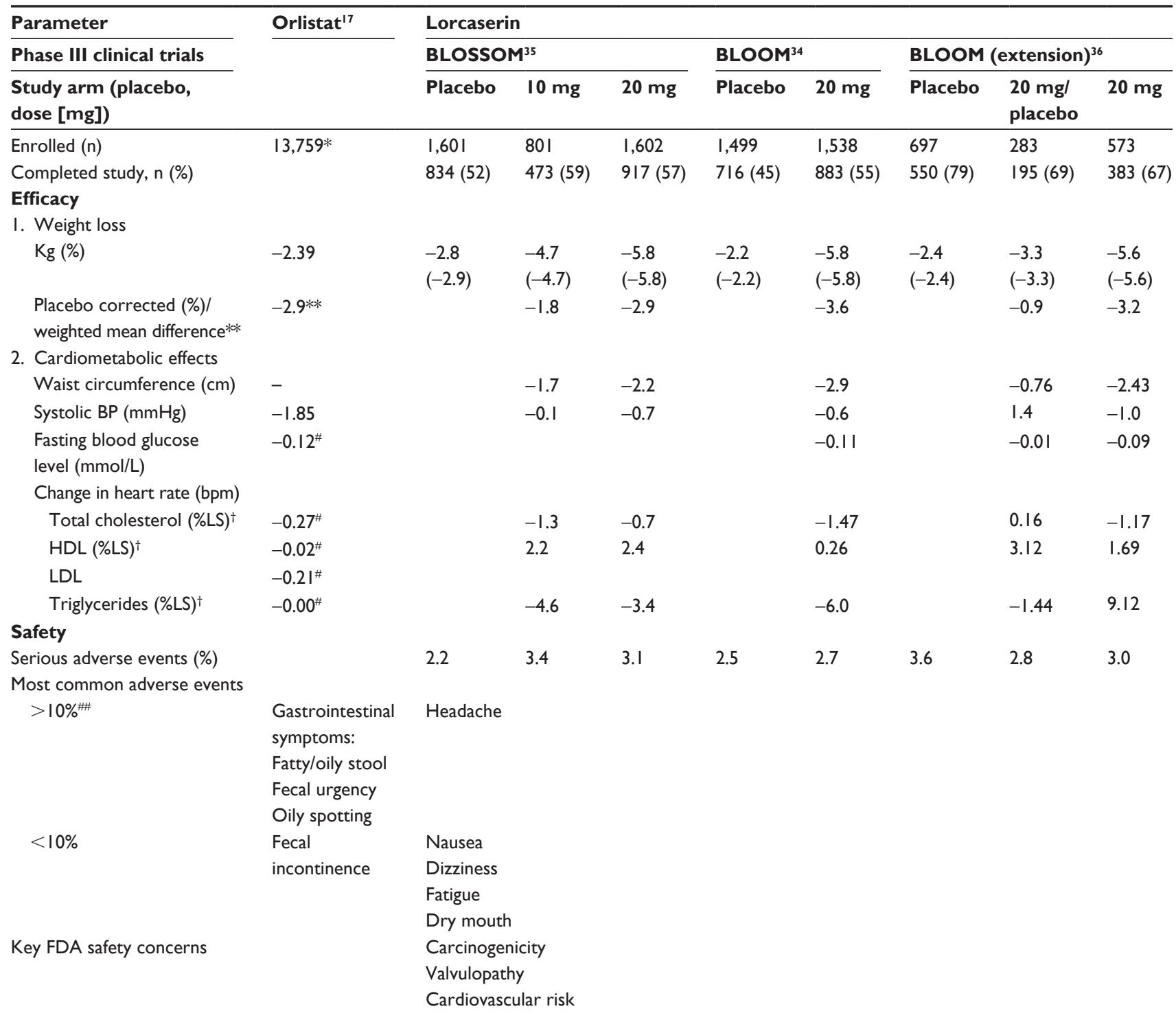

maintenance. This rationale is evident with the use of ER topiramate in phentermine/topiramate ER, enabling a longer duration of action with daily dosing, and improving tolerability compared to the immediate-release formulation.

Key safety considerations for phentermine/topiramate ER arise from the known adverse effect profiles of the constituent drugs, specifically potential teratogenicity, cardiovascular and neurocognitive sequelae, and metabolic acidosis. ${ }^{20}$ Accordingly, phentermine/topiramate ER is not recommended in individuals with recent/unstable vascular disease, significant active depression or suicidal ideation/previous suicide attempts, and it is contraindicated in those with glaucoma and hyperthyroidism. ${ }^{39}$

\section{Common adverse events}

Phentermine/topiramate ER appears to be generally well tolerated, as adverse events are predominantly dose-related and alleviated over time; this is evident with the significantly lower 


\begin{tabular}{|c|c|c|c|c|c|c|c|c|}
\hline \multicolumn{9}{|c|}{ Phentermine/topiramate ER } \\
\hline \multicolumn{3}{|c|}{ EQUATE $^{38,39}$} & \multicolumn{3}{|l|}{ EQUIP $^{41}$} & \multicolumn{3}{|c|}{ CONQUER $^{27}$} \\
\hline Placebo & $7.5 / 46 \mathrm{mg}$ & 15/92 mg & Placebo & $3.75 / 23 \mathrm{mg}$ & I5/92 mg & Placebo & $7.5 / 46 \mathrm{mg}$ & $15 / 92 \mathrm{mg}$ \\
\hline 103 & 103 & 103 & 514 & 241 & 512 & 994 & 498 & 995 \\
\hline $69(63)$ & $73(69)$ & $68(63)$ & $24 I(47)$ & $138(57)$ & $301(59)$ & $616(62)$ & $374(75)$ & $733(74)$ \\
\hline-1.5 & -8.4 & -8.9 & -1.8 & -6.0 & -12.6 & -1.4 & -8.1 & -10.2 \\
\hline \multirow[t]{9}{*}{$(-1.5)$} & $(-8.2)$ & $(-9.0)$ & $(-1.6)$ & $(-5.1)$ & $(-10.9)$ & $(-1.2)$ & $(-7.8)$ & $(-9.8)$ \\
\hline & -6.8 & -7.5 & & -3.5 & -9.3 & & -6.6 & -8.6 \\
\hline & -5.5 & -5.4 & & -2.5 & -7.8 & & -5.2 & -6.8 \\
\hline & -5.2 & -3.4 & & -2.7 & -3.8 & & -2.3 & -3.2 \\
\hline & 0.01 & -0.02 & & -0.06 & -0.14 & & -0.12 & -0.20 \\
\hline & & & -0.2 & -0.3 & 1.2 & -0.1 & 0.1 & 1.7 \\
\hline & -2.6 & -1.4 & & -1.9 & -2.5 & & -1.6 & -3 \\
\hline & 1.8 & 2.1 & & 0.5 & 3.5 & & 4 & 5.6 \\
\hline & -1.6 & -12.6 & & -3.9 & -14.3 & & -13.3 & -15.3 \\
\hline 3.5 & 3.0 & 4.0 & 2.5 & 2.5 & 2.5 & 4.0 & 3.0 & 5.0 \\
\hline \multicolumn{9}{|c|}{ Paresthesia } \\
\hline \multicolumn{9}{|c|}{ Dry mouth } \\
\hline \multicolumn{9}{|c|}{ Constipation } \\
\hline \multicolumn{9}{|l|}{ Dysgeusia } \\
\hline \multicolumn{9}{|l|}{ Dizziness } \\
\hline \multicolumn{9}{|l|}{ Insomnia } \\
\hline \multicolumn{9}{|l|}{ Nausea } \\
\hline \multicolumn{9}{|c|}{ Depression } \\
\hline \multicolumn{9}{|c|}{ Neurocognitive sequelae } \\
\hline \multicolumn{9}{|c|}{ Tachycardia } \\
\hline Birth defec & & & & & & & & \\
\hline
\end{tabular}

Notes: *Data from Zhou et al. ${ }^{48}$ Effect of anti-obesity drug on cardiovascular risk factors: A systematic review and meta-analysis of randomised controlled trials. PLoS One. 2012;7(6):e39062. Twenty-one RCTs met the inclusion criteria; **Data from Padwal et al. ${ }^{30}$ Long-term pharmacotherapy for obesity and overweight (Review). Cochrane

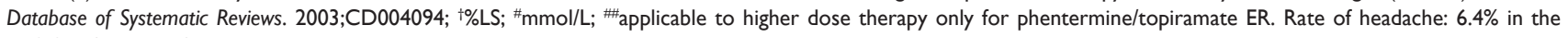
BLOOM (extension) study.

Abbreviations: ER, extended release; BLOSSOM, Behavioral Modification and Lorcaserin Second Study for Obesity Management; BLOOM, Behavioral Modification and Lorcaserin for Obesity and Overweight Management; n, number; BP, blood pressure; \%LS, percentage least squares; HDL, high-density lipoprotein; LDL, low-density lipoprotein; FDA, US Food and Drug Administration; RCT, randomized controlled trial.

incidence of adverse effects in the SEQUEL versus CONQUER studies. ${ }^{27,42}$ Common adverse events include paresthesia, dry mouth, dizziness, dysgeusia, headache, insomnia, and constipation. Discontinuation rates due to adverse events were $9 \%, 12 \%$, and $18 \%$ for placebo, low- and mid-dose, and highdose phentermine/topiramate ER, respectively. ${ }^{27,40,42}$ Overall, there was a higher study completion rate in those taking phentermine/topiramate ER compared with placebo, likely because of the greater weight loss efficacy of the intervention over placebo, and/or the effect of topiramate-mediated amelioration in food-addictive behavior. ${ }^{40}$

\section{Cardiovascular concerns}

The major concern regarding phentermine/topiramate ER relates to its possible deleterious effects on cardiovascular endpoints, as phentermine has been associated with an increase in heart rate. ${ }^{27,41}$ Evidence thus far indicates that this therapy is associated with only small increases in mean 
heart rate, with mid- and full-dose phentermine/topiramate ER associated with a mean increase in heart rate of $0.6 \mathrm{bpm}$ and $1.6 \mathrm{bpm}$, respectively, as compared with no change in the placebo-treated group. ${ }^{27,41}$ A greater proportion of individuals had a documented increase in heart rate when compared to placebo-treated patients, with $13.5 \%$ of individuals treated with the mid-dose, $19.6 \%$ with the full dose, and $11.9 \%$ on placebo reporting an increase in heart rate from baseline of $>20 \mathrm{bpm}^{27,41}$ The clinical significance of this is unclear; however, at this stage, this finding does not appear to be associated with any compromise in cardiac function and the changes in heart rate correlate with improvements in blood pressure parameters. ${ }^{27,41}$ Nevertheless, long-term cardiovascular safety data are required, particularly in individuals with more unstable cardiovascular disease, in view of the poor history of obesity pharmacotherapy characterized by significant adverse cardiovascular signals identified during post-marketing surveillance. Based on the interpretation of data in the SCOUT study, ${ }^{9}$ adverse cardiovascular events should be attenuated by appropriate patient selection for pharmacotherapy and continued pharmaco-vigilance, such that if a patient does not lose weight on a particular therapy, then that therapy should be discontinued.

\section{Neurocognitive sequelae}

Both phentermine and topiramate are associated with worsening mood disorders and suicidal ideation via central nervous system effects. ${ }^{27,28}$ To date, there is no evidence that combination pharmacotherapy significantly increases moderate/severe depression or suicidality in individuals with mild depression on stable doses of antidepressants. However, the clinical trials demonstrate that individuals receiving higher dose phentermine/topiramate ER compared to placebo were up to sevenfold more likely to cease therapy because of mental health-related effects. ${ }^{27,28}$

Topiramate is also associated with cognitive dysfunction, including language difficulties, attention and memory impairment, and confusion. 22,27,28 These effects were seen in individuals on phentermine/topiramate ER, with disturbances in attention occurring most often. The incidence of neurocognitive adverse effects was $2.0 \%$ for low-dose, $5.6 \%$ for mid-dose, and $7.8 \%$ for full-dose phentermine/topiramate ER compared to $1.7 \%$ for placebo. ${ }^{20}$

\section{Teratogenicity and contraception considerations}

The teratogenic potential of obesity pharmacotherapy is a major concern, as these agents are frequently used in women of childbearing potential. Moreover, topiramate may alter the pharmacokinetics of the oral contraceptive pill, with evidence suggesting that ethinyl estradiol levels may be decreased by $16 \%$, theoretically decreasing contraceptive efficacy. ${ }^{20}$ Accordingly, the FDA has developed guidelines to ensure that women of childbearing potential are informed of the possible teratogenic risks of the treatment, recommending that pregnancy testing be conducted prior to initiation of phentermine/topiramate ER, and suggesting that monthly pregnancy testing be performed thereafter. ${ }^{20}$ In addition, consistent use of effective contraception is essential.

FDA approval for phentermine/topiramate ER was contingent on an analysis of the potential risk of teratogenicity associated with its use, based on outcomes among infants born to mothers exposed to topiramate during pregnancy. ${ }^{44}$ The prevalence of oral clefts (cleft lip with or without cleft palate) was twofold greater in pregnancies exposed to topiramate, with an estimated prevalence rate among those actively using topiramate of $0.29 \%$, compared to $0.16 \%$ in previously exposed women. ${ }^{45,46}$

\section{Other adverse events}

Topiramate is associated with metabolic acidosis via carbonic anhydrase inhibition. ${ }^{46,47}$ Accordingly, a small decrease in serum bicarbonate concentration has been observed in all phentermine/topiramate ER studies without clinically significant metabolic acidosis, and serum bicarbonate levels tended to return to baseline over time. ${ }^{27,41,42}$ However, the paresthesia commonly seen with therapy is most likely associated with serum bicarbonate alterations. Chronic severe metabolic acidosis may lead to osteomalacia, osteoporosis, and renal calculi; ${ }^{47}$ however, these outcomes have not been seen in the trials to date.

\section{Conclusion}

Lifestyle interventions with adjunctive pharmacotherapy and bariatric surgery are the established approaches used to manage obesity and its cardiometabolic sequelae. Although lifestyle modification remains the cornerstone of obesity interventions, its effectiveness is frequently limited by significant weight regain in the long-term. Accordingly, successful obesity management requires escalation of intervention in many individuals, and adjunctive pharmacotherapy appears to be increasingly effective in this regard. It seems reasonable that pharmacotherapy be offered as an alternative intermediate option to lifestyle or bariatric surgery, expanding the current armamentarium 
of obesity management. The weight loss achieved by pharmacotherapy is in between that commonly achieved by lifestyle intervention and bariatric surgery. However, pharmacotherapy would ideally obviate the need for surgery in some individuals, providing it demonstrates a favorable cardiometabolic profile.

The burgeoning obesity epidemic arguably necessitates a paradigm shift in the current approach to obesity management, with greater consideration for the role of long-term pharmacotherapy to assist and maintain weight loss. Obesity must be conceptualized as a chronic disease associated with multiple comorbidities and, thus, any treatment approach must also be multifaceted and reflect the complex etiology of obesity.

Combination pharmacotherapy has theoretical appeal, as it better reflects the multiple pathophysiological mechanisms of obesity. Phentermine/topiramate ER thus currently demonstrates greater efficacy than monotherapy through its synergistic potential and multiple mechanisms of action, while its improved tolerability reinforces the role of combination pharmacotherapy in long-term weight loss maintenance. Current evidence suggests a positive, durable effect of phentermine/topiramate ER on the cardiometabolic sequelae of obesity, including improving glycemia and blood pressure, and ameliorating obstructive sleep apnea. More long-term efficacy and safety data are however required given the poor history of obesity pharmacotherapy.

\section{Disclosure}

The authors report no conflicts of interest in this work.

\section{References}

1. Finucane MM, Stevens GA, Cowan MJ, et al; Global Burden of Metabolic Risk Factors of Chronic Diseases Collaborating Group (Body Mass Index). National, regional, and global trends in body-mass index since 1980: systematic analysis of health examination surveys and epidemiological studies with 960 country-years and $9 \cdot 1$ million participants. Lancet. 2011;377(9765):557-567.

2. Vasan RS, Pencina MJ, Cobain M, Freiberg MS, D’Agostino RB. Estimated risks for developing obesity in the Framingham Heart Study. Ann Intern Med. 2005;143(7):473-480.

3. Kelly T, Yang W, Chen CS, Reynolds K, He J. Global burden of obesity in 2005 and projections to 2030. Int J Obes (Lond). 2008;32(9): 1431-1437.

4. Bleich SN, Bennett WL, Gudzune KA, Cooper LA. Impact of physician BMI on obesity care and beliefs. Obesity (Silver Spring). 2012;20(5): 999-1005.

5. Adams KF, Schatzkin A, Harris TB, et al. Overweight, obesity, and mortality in a large prospective cohort of persons 50 to 71 years old. N Engl J Med. 2006;355(8):763-778.

6. Whitlock G, Lewington S, Sherliker P, et al; Prospective Studies Collaboration. Body-mass index and cause-specific mortality in 900000 adults: collaborative analyses of 57 prospective studies. Lancet. 2009;373(9669):1083-1096.

7. Goldstein DJ. Beneficial health effects of modest weight loss. Int J Obes Relat Metab Disord. 1992;16(6):397-415.
8. Poirier P, Giles TD, Bray GA, et al; American Heart Association; Obesity Committee of the Council on Nutrition, Physical Activity, and Metabolism. Obesity and cardiovascular disease: pathophysiology, evaluation, and effect of weight loss: an update of the 1997 American Heart Association Scientific Statement on Obesity and Heart Disease from the Obesity Committee of the Council on Nutrition, Physical Activity, and Metabolism. Circulation. 2006;113(6):898-918.

9. James WP, Caterson ID, Coutinho W, et al; SCOUT Investigators. Effect of sibutramine on cardiovascular outcomes in overweight and obese subjects. N Engl J Med. 2010;363(10):905-917.

10. Mark EJ, Patalas ED, Chang HT, Evans RJ, Kessler SC. Fatal pulmonary hypertension associated with short-term use of fenfluramine and phentermine. N Engl J Med. 1997;337(9):602-606.

11. Connolly HM, Crary JL, McGoon MD, et al. Valvular heart disease associated with fenfluramine-phentermine. $N$ Engl J Med. 1997;337(9):581-588.

12. Yanovski SZ, Yanovski JA. Long-term drug treatment for obesity: a systematic and clinical review. JAMA. Epub November 14, 2013.

13. Bray GA, Ryan DH. Medical therapy for the patient with obesity. Circulation. 2012;125(13):1695-1703.

14. Lorello C, Goldfield GS, Doucet E. Methylphenidate hydrochloride increases energy expenditure in healthy adults. Obesity (Silver Spring). 2008;16(2):470-472.

15. Pasquali R, Casimirri F, Melchionda N, et al. Effects of chronic administration of ephidrine during very-low-calorie diets on energy expenditure, protein metabolism and hormone levels in obese subjects. Clin Sci (Lond). 1992;82(1):85-92.

16. Derosa G, Maffioli P. Anti-obesity drugs: a review about their effects and their safety. Expert Opin Drug Saf. 2012;11(3):459-471.

17. Li Z, Maglione M, Tu W, et al. Meta-analysis: pharmacologic treatment of obesity. Ann Intern Med. 2005;142(7):532-546.

18. Douglas A, Douglas JG, Robertson CE, Munro JF. Plasma phentermine levels, weight loss and side-effects. Int J Obes. 1983;7(6):591-595.

19. Hendricks EJ, Greenway FL. A study of abrupt phentermine cessation in patients in a weight management program. Am J Ther. 2011;18(4): 292-299.

20. Food and Drug Administration. Clinical Briefing Document, Endocrine and Metabolic Drugs Advisory Committee Meeting, February 22, 2012. New Drug Application 22580: VI-052 Qnexa (phentermine/topiramate). Silver Spring, MD: Food and Drug Administration; 2012 Available from: http:/www.fda.gov/downloads/AdvisoryCommittees/CommitteesMeetingMaterials/Drugs/EndocrinologicandMetabolicDrugsAdvisoryCommittee/UCM292315.pdf. Accessed July 3, 2013.

21. Braga MF, Aroniadou-Anderjaska V, Li H, Rogawski MA. Topiramate reduces excitability in the basolateral amygdala by selectively inhibiting GluK1 (GluR5) kainate receptors on interneurons and positively modulating GABAA receptors on principal neurons. J Pharmacol Exp Ther. 2009;330(2):558-566.

22. Verrotti A, Scaparrotta A, Agostinelli S, Di Pillo S, Chiarelli F, Grosso S. Topiramate-induced weight loss: a review. Epilepsy Res. 2011;95(3):189-199.

23. Picard F, Deshaies Y, Lalonde J, Samson P, Richard D. Topiramate reduces energy and fat gains in lean $(\mathrm{Fa} /$ ?) and obese (fa/fa) Zucker rats. Obes Res. 2000;8(9):656-663.

24. Tonstad S, Tykarski A, Weissgarten J, et al. Efficacy and safety of topiramate in the treatment of obese subjects with essential hypertension. Am J Cardiol. 2005;96(2):243-251.

25. Shank RP, Gardocki JF, Streeter AJ, Maryanoff BE. An overview of the preclinical aspects of topiramate: pharmacology, pharmacokinetics, and mechanism of action. Epilepsia. 2000;41 Suppl 1:S3-S9.

26. Ben-Menachem E, Axelsen M, Johanson EH, Stagge A, Smith U. Predictors of weight loss in adults with topiramate-treated epilepsy. Obes Res. 2003;11(4):556-562.

27. Gadde KM, Allison DB, Ryan DH, et al. Effects of low-dose, controlled-release, phentermine plus topiramate combination on weight and associated comorbidities in overweight and obese adults (CONQUER): a randomised, placebo-controlled, phase 3 trial. Lancet. 2011;377(9774):1341-1352. 
28. Brandes JL, Saper JR, Diamond M, et al; MIGR-002 Study Group. Topiramate for migraine prevention: a randomized controlled trial. JAMA. 2004;291(8):965-973.

29. Henry RR, Chilton R, Garvey WT. New options for the treatment of obesity and type 2 diabetes mellitus (narrative review). J Diabetes Complications. 2013;27(5):508-518.

30. Padwal R, Li SK, Lau DC. Long-term pharmacotherapy for overweight and obesity: a systematic review and meta-analysis of randomized controlled trials. Int J Obes Relat Metab Disord. 2003;27(12): 1437-1446.

31. Torgerson JS, Hauptman J, Boldrin MN, Sjöström L. XENical in the prevention of diabetes in obese subjects (XENDOS) study: a randomized study of orlistat as an adjunct to lifestyle changes for the prevention of type 2 diabetes in obese patients. Diabetes Care. 2004;27(1):155-161.

32. Rössner S, Sjöström L, Noack R, Meinders AE, Noseda G. Weight loss, weight maintenance, and improved cardiovascular risk factors after 2 years treatment with orlistat for obesity. European Orlistat Obesity Study Group. Obes Res. 2000;8(1):49-61.

33. Bays HE. Lorcaserin and adiposopathy: 5-HT2c agonism as a treatment for 'sick fat' and metabolic disease. Expert Rev Cardiovasc Ther. 2009;7(11):1429-1445.

34. Smith SR, Weissman NJ, Anderson CM, et al; Behavioral Modification and Lorcaserin for Overweight and Obesity Management (BLOOM) Study Group. Multicenter, placebo-controlled trial of lorcaserin for weight management. N Engl J Med. 2010;363(3):245-256.

35. Fidler MC, Sanchez M, Raether B, et al; BLOSSOM Clinical Trial Group. A one-year randomized trial of lorcaserin for weight loss in obese and overweight adults: the BLOSSOM trial. J Clin Endocrinol Metab. 2011;96(10):3067-3077.

36. O'Neil PM, Smith SR, Weissman NJ, et al. Randomized placebocontrolled clinical trial of lorcaserin for weight loss in type 2 diabetes mellitus: the BLOOM-DM study. Obesity (Silver Spring). 2012;20(7): 1426-1436.

37. Wyatt HR. Update on treatment strategies for obesity. J Clin Endocrinol Metab. 2013;98(4):1299-1306.

38. Ryan D, Peterson C, Troupin B, et al. Weight loss at 6 months with VI-0521 (PHEN/TPM combination) treatment. Obes Facts 2010:3146.
39. Robert M. Clinical Briefing Document. Endocrinologic and Metabolic Drugs Advisory Committee Meeting for phentermine/topiramate (Qnexa). Available from: http://www.fda.gov/downloads/AdvisoryCommittees/CommitteesMeetingsMaterials/Drugs/EndocrinologicandMetabolicDrugsAdvisoryCommittee/UCM218824.pdf (2010). Accessed August 9, 2013.

40. Bays H. Phentermine, topiramate and their combination for the treatment of adiposopathy ('sick fat') and metabolic disease. Expert Rev Cardiovasc Ther. 2010;8(12):1777-1801.

41. Allison DB, Gadde KM, Garvey WT, et al. Controlled-release phentermine/topiramate in severely obese adults: a randomized controlled trial (EQUIP). Obesity (Silver Spring). 2012;20(2):330-342.

42. Garvey WT, Ryan DH, Look M, et al. Two-year sustained weight loss and metabolic benefits with controlled-release phentermine/ topiramate in obese and overweight adults (SEQUEL): a randomized, placebo-controlled, phase 3 extension study. Am J Clin Nutr. 2012; 95(2):297-308

43. Winslow DH, Bowden CH, DiDonato KP, McCullough PA. A randomized, double-blind, placebo-controlled study of an oral, extended-release formulation of phentermine/topiramate for the treatment of obstructive sleep apnea in obese adults. Sleep. 2012;35(11):1529-1539.

44. Margulis AV, Mitchell AA, Gilboa SM, et al. National birth defects prevention study. Use of topiramate in pregnancy and risk of oral clefts. Am J Obstet Gynecol. 2012;207:405.e1-e7.

45. Hunt S, Russell A, Smithson WH, et al; UK Epilepsy and Pregnancy Register. Topiramate in pregnancy: preliminary experience from the UK Epilepsy and Pregnancy Register. Neurology. 2008;71(4): 272-276.

46. Mirza N, Marson AG, Pirmohamed M. Effect of topiramate on acid-base balance: extent, mechanism and effects. Br J Clin Pharmacol. 2009; 68(5):655-661.

47. Epstein DL, Grant WM. Carbonic anhydrase inhibitor side effects. Serum chemical analysis. Arch Ophthalmol. 1977;95(8):1378-1382.

48. Zhou YH et al. Effect of anti-obesity drug on cardiovascular risk factors: A systematic review and meta-analysis of randomised controlled trials. PLoS One. 2012;7(6):e39062.

49. Rueda-Clausen CF, Padwal RS, Sharma AM. New pharmacological approaches for obesity management. Nat Rev Endocrinol. 2013;9:467-478.

\section{Publish your work in this journal}

Diabetes, Metabolic Syndrome and Obesity: Targets and Therapy is an international, peer-reviewed open-access journal committed to the rapid publication of the latest laboratory and clinical findings in the fields of diabetes, metabolic syndrome and obesity research. Original research, review, case reports, hypothesis formation, expert opinion and commentaries are all considered for publication. The manuscript management system is completely online and includes a very quick and fair peer-review system, which is all easy to use. Visit http://www.dovepress.com/testimonials.php to read real quotes from published authors. 